\title{
Current Situation and Challenges Surrounding the Communication Gap Among Health-care Professionals: Outlook of Rural Health-care Professionals and Medical Students
}

\author{
Yoshihisa Hirakawa \\ Center for Postgraduate Clinical Training and Career Development, Nagoya University Hospital, Japan
}

(J Rural Med 2014; 9(1): 43-44)

\begin{abstract}
How well health-care professionals understand the positions of their colleagues and communicate with one another greatly impacts the success of community care ${ }^{1)}$. However, few studies on the topic have been conducted, especially in rural areas. Therefore, we organized a one-hour multidisciplinary group discussion on the topic of interdisciplinary collaboration in August 2013 in Shinshiro, a city located in a rural and remote area. The discussion was attended by health professionals including doctors and nurses working at clinics or community hospitals in a remote area. Medical students from two different medical schools also participated in the discussion. Fifty-five participants (15 doctors, 7 nurses, 5 hospital clerks, 3 medical social workers, 2 comedical staff, 1 pharmacist, 1 public health nurse, and 21 medical students) were recruited. The participants were randomly placed into 7 groups of 7 or 8 participants. They were instructed to write down all of the information formulated during the discussion. Following the discussion, the authors made a list of all the ideas or thoughts that emerged from the various groups based upon the participants' written notes; they then transferred all these ideas onto individual labels. A total of 319 labels were compiled reflecting the perceptions of the health-care professionals and medical students toward the communication gap among health-care professionals. We used the $\mathrm{KJ}$ method as a qualitative research tool $^{2,3)}$. First, to make it possible to quickly and efficiently frame key concepts into labels, we selected 35 of the 319 labels. Second, we organized the 35 labels into groups and combined labels that shared strong similarity in terms of quality as a result of grouping. Finally, we arranged these 10 groups according to the interrelationships among the groups (Figure 1).
\end{abstract}

Correspondence: Yoshihisa Hirakawa, Center for Postgraduate Clinical Training and Career Development, Nagoya University Hospital, 65 Tsuruma-cho,Showa-ku,Nagoya City, Aichi 466-8560, Japan

E-maily.hirakawa@med.nagoya-u.ac.jp
Fortunately, a number of measures can be adopted to break down these walls and revitalize communication. First, while doctors tend to feel superior in rank to other professionals, they should strive to communicate more efficiently with them; as well, other professionals should not hesitate to consult doctors when doubt arises. Second, the care team should preferably be lead by a professional other than a doctor. In order to lead the care team effectively, this professional needs to take pride in his/her work and contribution so as to earn the trust of doctors and other care team members. Third, mediators or facilitators are needed to help promote and monitor multidisciplinary relationships. Fourth, the care team should avoid the use of abbreviations or technical terms that often intimidate nonmedical staff; additionally, workplace courtesy should be encouraged through proper greetings and light conversation, and whenever possible, face-toface communication should prevail over indirect modes of communication such as the telephone, (electronic) medical records, or messages. This is often more easily achieved at clinics than at large hospitals. Finally, we should promote exchange among professionals both during and outside working hours to encourage teamwork and shared objectives.

Thus, our results offer insight on ways to bridge the communication barriers among health-care professionals in the community.

\section{References}

1. Shimada C, Takahashi R. [Multidisciplinary cooperation in the process of end of life care among older people]. Nippon Ronen Igakkai Zasshi Japanese Journal of Geriatrics 2011; 48: 221-226 (in Japanese). [Medline] [CrossRef]

2. Raymond S. The KJ Method: A Technique for Analyzing Data Derived from Japanese Ethnology. Hum Organ 1997; 56: 233-237.

3. Hirakawa Y, Uemura K. A qualitative study of long-term care leaders' experiences of end-of-life care provision at long-term care facilities in Japan. J Nurs Care 2013; S5: 010. 


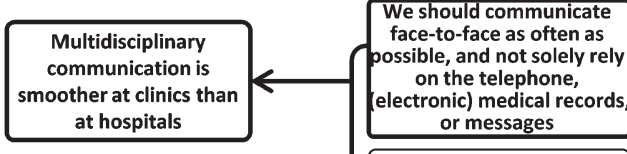

We should communicate face-to-face as often as possible

Messy handwriting on medical records is a barrier to multidisciplinary communication

While doctors should professiate with oth professionals more efficiently, other professionals should doubt arises

Doctors should recognize the barriers to multidisciplinary communication and pay attention to the opinion of other professionals

Some doctors do not want

to accept the opinion of other professionals

Doctors should

acknowledge the opinion of other professionals

While inter-disciplinary

interactions amon

professionals are rarely

smooth, they are not as

strained as with doctors

which often lead to multidisciplinary

communication failure

Doctors are often

conscious of the

bartiers to

communication

Co-medical staff should

consult a doctor when a doubt arises

Doctors (who tend to be in a higher position) should communicate with co-medical staff more efficiently

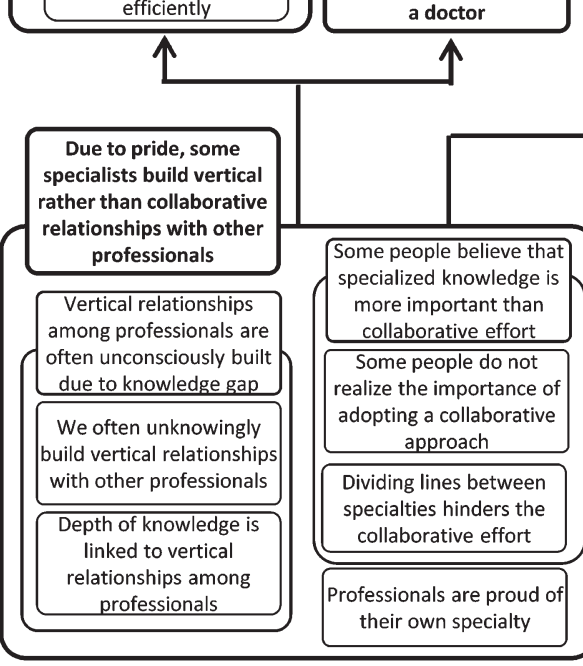

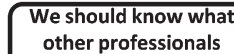

expect of us and assum

responsibility for our

own work to gain their trust

We should know what ther professionals expect of us

It is important for us to

own work in order to

gain the trust of other

professionals

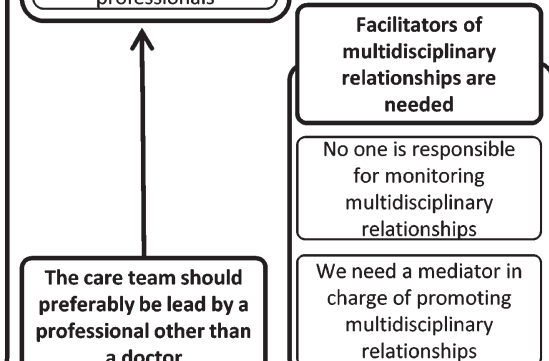

ships
Because our exchanges are

telephone, we have

superficial relationships

with professionals in our community

Electronic medical records

are a barrier to face-to-face

multidisciplinary

communication

Some people communicate only with department heads

and avoid contact with the staff

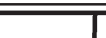

.

Greetings and light

conversation should be

encouraged to promote

multidisciplinary

collaboration

Daily conversation with other professionals

promotes

multidisciplinary

understanding

We need professional

exchanges as well as light

conversation to promote multidisciplinary conversation

Frequent use of erms hinders communication

Greetings are a key

element of success

multidisciplinary collaboration abbreviations or technical

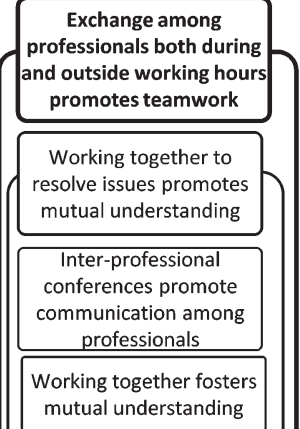

promotes mutual understanding among professionals

Common topics such as clients promote multidisciplinary communication

To appreciate the position of other professionals, it is necessary to fully understand their work and responsibilities

To appreciate the position of other professionals, it is important to fully responsibilities an responsibilities

Practical experience allowing insight into othe included in school curriculum curriculum

multidisciplinary events

should be held to promote mutual understanding 